\title{
Clinical experience of robotic myomectomy for fertility preservation using preoperative magnetic resonance imaging predictor
}

So Yun Park, MD, PhD ${ }^{1, *}$, Juhui Kim, $M D^{2,{ }^{2}}$, Kyungah Jeong, $M D, P h D^{2}$, Sung II Jung, MD, PhD ${ }^{3}$, Young Min Hur, MD'2, Eun Hye Cho, MD², Hye-Sung Moon, MD, PhD', Hye Won Chung, MD, PhD

Department of Obstetrics and Gynecology, ${ }^{1}$ Ewha Womans University Seoul Hospital, ${ }^{2}$ Ewha Womans University Mokdong Hospital, College of Medicine, Ewha Womans University; ${ }^{3}$ Department of Radiology, Konkuk University Medical Center, Research Institute of Medical Science, Konkuk University School of Medicine, Seoul, Korea

\section{Objective}

This study aimed to demonstrate the use of preoperative magnetic resonance imaging (MRI) findings to select the optimal surgical technique between single-site (SS) and multi-site (MS) robotic myomectomy based on clinical experience, for the preservation of fertility.

\section{Methods}

Ninety-eight patients who underwent SS or MS robotic myomectomy using the da Vinci ${ }^{\circledR}$ Si system after undergoing MRI were evaluated retrospectively. The correlation between preoperative MRI findings and the intraoperative or postoperative findings during robotic myomectomy for the preservation of fertility was analyzed. The reproductive outcome was investigated when the patient wished to conceive.

\section{Results}

The mean age of the patients was $35.68 \pm 5.04$ years and 80 patients $(81.6 \%)$ were nulliparous. The total diameter of myomas on MRI was $106.75 \pm 54.52 \mathrm{~mm}$. The number of resected myomas was $4.31 \pm 4.39$ (range, 1-27), and the total weight of resected myomas was $293.11 \pm 281.13$ (range, 30-1,260) g. Myomas with high signal intensity on MRI required less time for resection. MS robotic myomectomy was performed for an increased number and total diameter of a myoma or a deep-seated myoma. Postoperatively, all patients resumed normal menstruation. Of the 15 patients who wished to conceive, $12(80 \%)$ conceived successfully. Of these, uterine dehiscence occurred in 1 patient and 10 patients underwent an uneventful cesarean section.

\section{Conclusion}

SS or MS robotic myomectomy can be recommended for patients who wish to conserve fertility. However, the optimal surgical technique should be selected based on preoperative MRI findings to predict an effective surgical process and the successful preservation of fertility.

Keywords: Uterine myomectomy; Magnetic resonance imaging; Fertility preservation

\section{Introduction}

Uterine leiomyoma, which originates from the smooth muscle cells of the uterus, is a common benign tumor among women of reproductive age. Myomas are associated with symptoms such as prolonged or heavy menstrual bleeding, pelvic pressure or pain, and reproductive dysfunction.

Received: 2020.05.31. Revised: 2020.08.03. Accepted: 2020.08.10. Corresponding author: Kyungah Jeong, $\mathrm{MD}, \mathrm{PhD}$

Department of Obstetrics and Gynecology, Ewha Womans University Mokdong Hospital, College of Medicine, Ewha Womans University, 1071 Anyangcheon-ro, Yangcheon-gu, Seoul 07985, Korea

E-mail: ogjeong@ewha.ac.kr

https://orcid.org/0000-0002-9673-1152

*These authors contributed equally to this work.

Articles published in Obstet Gynecol Sci are open-access, distributed under the terms of the Creative Commons Attribution Non-Commercial License (http://creativecommons. org/licenses/by-nc/3.0/) which permits unrestricted non-commercial use, distribution, and reproduction in any medium, provided the original work is properly cited.

Copyright $\odot 2020$ Korean Society of Obstetrics and Gynecology 


\section{Obstetrics \& Gynecology Science}

So Yun Park, et al. Robotic myomectomy using MRI predictor

Myomas are clinically evident in approximately $25 \%$ of the women who start manifesting symptoms in their 30s or 40s [1].

Due to a shift in the social trend towards delayed child bearing in recent times, further knowledge about the association between fertility and myomas has become necessary. A recent cohort study revealed that myomas have a negative effect on fertility and lead to delayed conception [2]. The proportion of young women with myoma who require pertinent treatment for future pregnancy has increased significantly.

Myomectomy is usually the treatment of choice for women with unexplained infertility other than for those with the presence of myomas. Although an appropriate minimal invasive surgical technique is recommended, laparoscopic myomectomy has some systemic limitations. With advancements in surgical techniques, it has become possible to overcome the disadvantages associated with conventional laparoscopic surgery. The da $\operatorname{Vinci}^{\circledR}$ Si system (Intuitive Surgical, Inc., Sunnyvale, CA, USA) provides a stable 3-dimensional vision, instruments with a wrist function, tremor elimination, greater precision in dissection, easier knot-and-tying, and favorable ergonomics [3].

If the operative outcomes turn out to be similar, young women hope to decrease the sizes and the numbers of their scars without a doubt. However, single-port laparoscopy is inconvenient for most surgeons due to the non-optimal or limited surgical field because of parallel movement of the rigid surgical instruments from one direction of the umbilicus. Robotic single-site (SS) surgery was developed and used in clinical settings, because it causes less pain and has a higher patient satisfaction. The da Vinci ${ }^{\circledR}$ Si system, which was used for SS robotic surgery, has some disadvantages including an unfamiliar docking process, non-articulated semi-rigid instruments, and the lack of monopolar scissors [4]. Therefore, it is essential to select the suitable patient group while performing SS robotic surgery.

Pelvic ultrasonography through the vaginal approach is the most commonly used diagnostic method for identifying the location or shape of the uterine myoma. However, magnetic resonance imaging (MRI) is superior to transvaginal ultrasonography in ascertaining both, the embedment into the uterine wall, and the exact size and position of myomas [5]. Moreover, fibroid stiffness can be gauged from other T2 imaging characteristics, e.g., fibroids that appear bright on T2 imaging are less stiffer than those that appear dark [6]. Therefore, surgeons were able to customize the surgical technique to preserve the fertility, after introduction of the concept of preoperative MRI.

We aimed to demonstrate the usefulness of preoperative MRI to select the optimal surgical technique between SS and multi-site (MS) robotic myomectomy to preserve fertility, based on the clinical experience of robotic myomectomy.

\section{Materials and methods}

A retrospective chart review of 98 patients who underwent MRI before robotic myomectomy using the da $\operatorname{Vinci}^{\circledR} \mathrm{Si}$ system from July 2015 to March 2018 at Ewha Womans University Mokdong Hospital was performed. Before the surgery, pelvic MRI scans were obtained using the 3.0-T scanner (Achieva; Philips Medical Systems, Cleveland, OH, USA) using a pelvic array coil. SS or MS robotic myomectomy was performed on all patients by 2 surgeons who had an experience of performing more than 100 gynecologic robotic surgeries and about 2,000 laparoscopic surgeries. All patients who underwent MRI before robotic myomectomy by the 2 surgeons were enrolled without any exclusion. The final pathology was confirmed as leiomyoma. The preoperative MRI findings, and intraoperative or postoperative findings during robotic myomectomy for the preservation of fertility were analyzed.

A radiologist with 15 years of experience in gynecologic imaging retrospectively reviewed all MRI scans on a picture archiving and communication system workstation (Centricity; GE Healthcare, Milwaukee, WI, USA). Based on the myoma sub-classification system by the International Federation of Gynecology and Obstetrics (FIGO), the number (diameter, $<3 \mathrm{~cm}$ ), size, signal intensity, enhancement (E) of myoma, endometrial distortion, thickness of the endometrium, and junctional zone were assessed from the MRI scans.

The duration of surgery, estimated blood loss (EBL), time required for the resection of the myoma, and the number and total weight of the resected myoma were measured. After 2 months, postoperative pelvic ultrasonography was performed to define the shape of the uterus as well as to check the endometrial thickness and pattern. The reproductive outcomes were investigated when the patients wished to conceive.

Statistical analysis was performed using SPSS version 20 (IBM, Seoul, Korea). The categorical variables were presented as numbers and percentages, and the normally distributed 


\title{
Obstetrics \& Gynecology Science
}

\author{
Vol. 63, No. 6,2020
}

continuous variables were presented as the mean and the standard deviation, with range information. Data were analyzed for statistical significance with a one-way analysis of variance, using the student's $t$-test and Mann-Whitney $U$ test. The correlations between the findings on preoperative MRI and intraoperative or postoperative findings were analyzed using Pearson's correlation coefficient. $P$-values $<0.05$ were considered statistically significant.

\section{Results}

Mean age of patients in the study was $35.68 \pm 5.04$ (range, $23-45)$ years, and 80 patients ( $81.6 \%$ ) were nulliparous. The preoperative MRI findings of 98 subjects were described, and compared between the group that underwent SS and the group that underwent MS robotic myomectomy (Table 1). As observed on MRI scans, the total diameter of the myomas was $106.75 \pm 54.52$ (range, 32.43-273.24) $\mathrm{mm}$, while the maximum diameter was $72.51 \pm 25.77$ (range, 32.43-151.03) $\mathrm{mm}$. Furthermore, the endometrial thickness was $6.44 \pm 3.81$ $\mathrm{mm}$ and the endometrium was distorted in $70.4 \%$ of the patients.

The intraoperative findings during robotic myomectomy and the comparison between SS and MS groups are shown in Table 2. The duration of surgery was $157.89 \pm 61.22$ (range, 60-355) $\mathrm{min}$, and the time taken for resection was $13.83 \pm 17.31$ (range, 1-90) minutes. The number of resected myomas was $4.31 \pm 4.39$ (range, 1-27), and their total weight was 293.11 \pm 281.13 (range, 30-1,260) g. Compared to SS robotic myomectomy, conventional MS robotic myomectomy was performed when the preoperative number and the total diameter of myomas were high or if the myomas were deepseated according to the FIGO classification. The location of myomas by the FIGO classification was significantly different between the SS and MS groups, when analyzed using the Mann-Whitney $U$ test (median 4 in SS group vs. median 3 in MS group, $P$ for trend=0.001).

The preoperative total diameter of the myoma on MRI was significantly correlated with the duration of surgery, EBL, and time required for the resection of the myoma. Fifteen patients (15.3\%) required blood transfusion, and their EBL and resection time were significantly higher. Post-operative complications were seen in 7 cases. There were 3 cases of postoperative fever, and one case each of post-operative ileus, small bowel perforation, and ureteral injury. Six patients with complications recovered after hydration therapy, antibiotic administration, primary repair, and ureteral stent insertion.

Table 1. Comparison of preoperative magnetic resonance imaging (MRI) findings between single-site (SS) and multi-site (MS) robotic myomectomy

\begin{tabular}{lcccc}
\hline MRI findings & Mean \pm SD (range) & SS $(\mathbf{n = 4 3 )}$ & MS (n=55) & $\boldsymbol{P}$-value \\
\hline No. of myoma $(>3 \mathrm{~cm})$ & $1.95 \pm 1.22(1-7)$ & $1.65 \pm 1.21$ & $2.18 \pm 1.19$ & NS \\
Total diameter of myoma $(\mathrm{mm})$ & $106.75 \pm 54.52(32.43-273.24)$ & $81.69 \pm 42.43$ & $125.43 \pm 55.35$ & 0.0001 \\
Maximum diameter of myoma $(\mathrm{mm})$ & $72.51 \pm 25.77(32.43-151.03)$ & $63.31 \pm 20.57$ & $79.28 \pm 27.26$ & 0.03 \\
Endometrial thickness $(\mathrm{mm})$ & $6.44 \pm 3.81(1.29-21.24)$ & $6.94 \pm 3.62$ & $6.05 \pm 3.94$ & $\mathrm{NS}$ \\
Junctional zone thickness $(\mathrm{mm})$ & $3.67 \pm 1.82(1.16-14.59)$ & $3.94 \pm 2.19$ & $3.45 \pm 1.45$ & NS \\
\hline
\end{tabular}

SD, standard deviation; NS, non-specific.

Table 2. Comparison of operative findings between single-site (SS) and multi-site (MS) robotic myomectomy

\begin{tabular}{lcccc}
\hline Operative findings & Mean \pm SD (range) & SS $(\mathbf{n = 4 3 )}$ & MS (n=55) & $\boldsymbol{P}$-value \\
\hline Operation time $(\mathrm{min})$ & $157.89 \pm 61.22(60-355)$ & $129.42 \pm 47.07$ & $180.56 \pm 62.05$ & 0.0001 \\
Estimated blood loss $(\mathrm{mL})$ & $255.92 \pm 213.44(20-1,050)$ & $174.19 \pm 160.26$ & $319.82 \pm 228.87$ & 0.001 \\
Chopping time $(\mathrm{min})$ & $13.83 \pm 17.31(1-90)$ & $6.83 \pm 7.26$ & $18.15 \pm 20.17$ & 0.001 \\
No. of removed myoma & $4.31 \pm 4.39(1-27)$ & $2.95 \pm 2.35$ & $5.36 \pm 5.27$ & 0.003 \\
Total weight of removed myoma (g) & $293.11 \pm 281.13(30-1,260)$ & $187.38 \pm 165.51$ & $360.78 \pm 318.35$ & 0.002 \\
\hline
\end{tabular}

SD, standard deviation. 


\section{Obstetrics \& Gynecology Science}

So Yun Park, et al. Robotic myomectomy using MRI predictor

Uterine dehiscence occurred in one patient during pregnancy (Table 3).

Myomas with a low T2 signal intensity were considerably stiffer, which thereby prolonged their resection time (Fig. 1). Between patients who had signal intensity 1 on $\mathrm{T} 2$ and those who had intensity 3 , the mean time required for resection was significantly different $(14.87 \pm 17.99$ vs. $7.75 \pm 6.71 \mathrm{~min}-$ utes, $P=0.046)$.

The mean EBL (E1: $246.33 \pm 225.63, E 2: 254.00 \pm 178.52$, E3: $273.33 \pm 254.01 \mathrm{~mL}$ ) during surgery was also high when the vascularity was high and the myoma was strongly enhanced on the T1 image; however, the P-value was not statistically significant (Fig. 2).

After robotic myomectomy, all patients resumed normal menstruation and showed the normal shape of the uterus (91.8\%), as well as normal endometrial thickness (mean, $8.53 \pm 3.52 \mathrm{~mm}$; range, $1.8-19.73 \mathrm{~mm}$ ) and pattern $(98.0 \%)$ on pelvic ultrasound scans, although $70.4 \%$ of the patients had a compressed or distorted endometrium. Pearson correlation coefficient showed a negative correlation between the maximum diameter of myoma and postoperative endometrial thickness although it was not statistically significant $(R=-0.139 ; P=0.195)$. There were no significant differences in postoperative outcomes based on the preoperative MRI findings or the robotic surgical technique (SS vs. MS) used.

Among 15 patients who wished to conceive, 12 (80\%) conceived successfully. While 10 of these patients conceived naturally, 2 patients conceived through assisted reproduc-

Table 3. Complications of robotic myomectomy

\begin{tabular}{lccc}
\hline Complications & No. of patients & Surgical technique & Characteristics \\
\hline $\begin{array}{l}\text { Intra-operative } \\
\text { Small bowel perforation }\end{array}$ & 1 & MS & $\begin{array}{c}\text { Myoma } 1,180 \mathrm{~g} \\
\text { Previous C/S }\end{array}$ \\
Ureteral injury & 1 & MS & Combined advanced endometriosis \\
$\begin{array}{l}\text { Post-operative } \\
\text { Ileus }\end{array}$ & 1 & $\mathrm{SS}$ & Previous C/S \\
Fever & 3 & SS (2), MS (1) & Clostridium difficile \\
Obstetric & 1 & MS & 27.1 weeks of pregnancy, male $963 \mathrm{~g}$, neonatal death \\
Uterine dehiscence
\end{tabular}

SS, single-site; MS, multi-site; C/S, caesarean section.

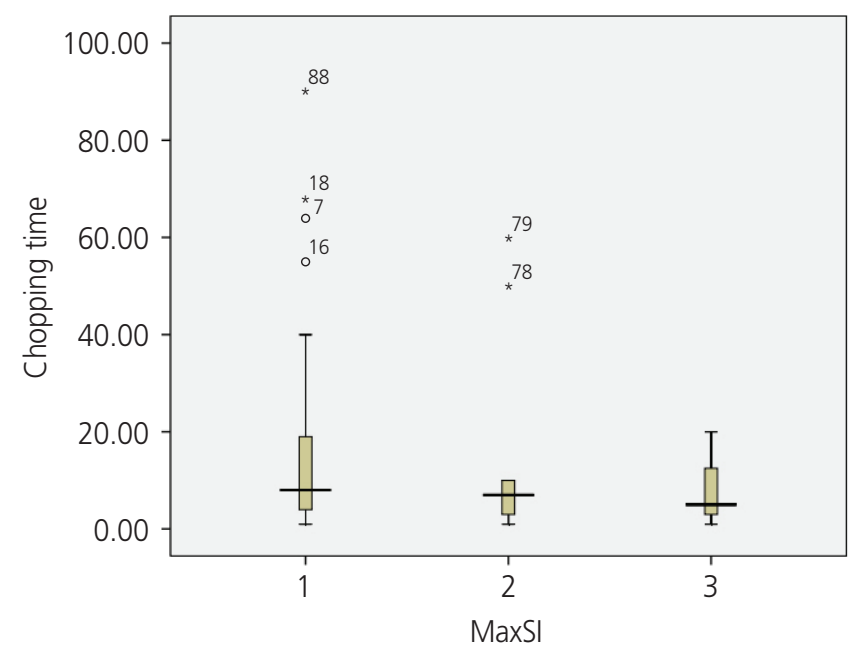

Fig. 1. The relationship between $\mathrm{T} 2$ signal intensity (SI) and chopping time.

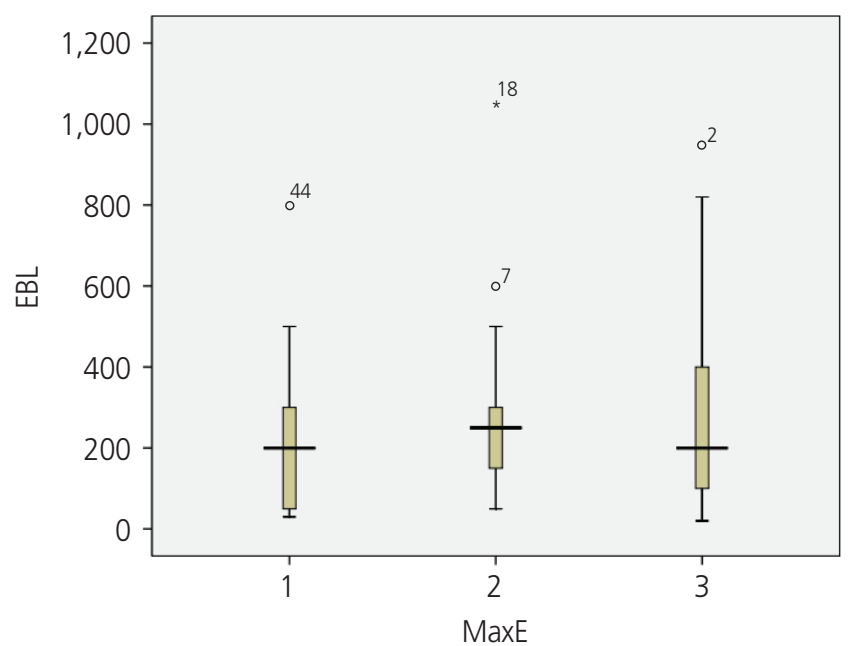

Fig. 2. The relationship between enhancement and estimated blood loss (EBL). 


\section{Obstetrics \& Gynecology Science}

Vol. 63, No. 6, 2020

tion techniques. One patient who underwent intrauterine insemination after controlled ovarian stimulation successfully delivered healthy triplets by cesarean section. The preoperative MRI and intraoperative pictures of this case are shown in Fig. 3. A patient who had an unsuccessful in vitro fertilization before undergoing robotic myomectomy, successfully conceived with thawing embryo transfer, after robotic myomectomy was performed. One patient experienced spontaneous abortion. Unfortunately, uterine dehiscence occurred at in a woman at 27.1 weeks of pregnancy; she had a thin fundus (6.5-mm). She had undergone robotic myomectomy for a huge myoma (total weight: 1,080 g) with endometrial distortion. The remaining 10 patients underwent an uneventful cesarean section at term.

\section{Discussion}

Few papers have been published on the feasibility of robotic myomectomy for the preservation of fertility, especially including a comparison between SS and MS robotic myomectomy procedures. In this study, we additionally aimed to demonstrate the usefulness of preoperative MRI findings to select the optimal surgical technique between SS and MS.
Wang et al. [7] compared the outcomes of patients who underwent abdominal myomectomy, laparoscopic myomectomy, and robotic myomectomy. They reported that robot-assisted myomectomy had fewer postoperative complications, lesser blood loss during surgery, and lesser pain after surgery compared to laparoscopic myomectomy or abdominal myomectomy.

Our study corroborates the findings of recent studies, which reported that robotic myomectomy is a good choice for patients who seek preservation of fertility and has fewer complications. Huberlant et al. [8] published a retrospective study comprising a series of 53 patients who underwent robotic myomectomy over 7 years in a single institution. They reported the advantages of this robotic technique for women with infertility, because it has a low rate of obstetrical complications, with no risk of uterine rupture and abnormal placenta, with fewer intrauterine adhesions as opposed to the complications associated with laparoscopic myomectomy or abdominal myomectomy. A large-scale study on long-term outcomes, including the results of pregnancy, was conducted among 852 women who underwent robotic myomectomy. It was found that $62.9 \%$ of the women remained symptomfree $>3$ years after undergoing robotic myomectomy, and $50.8 \%$ of women desirous of pregnancy were able to con-
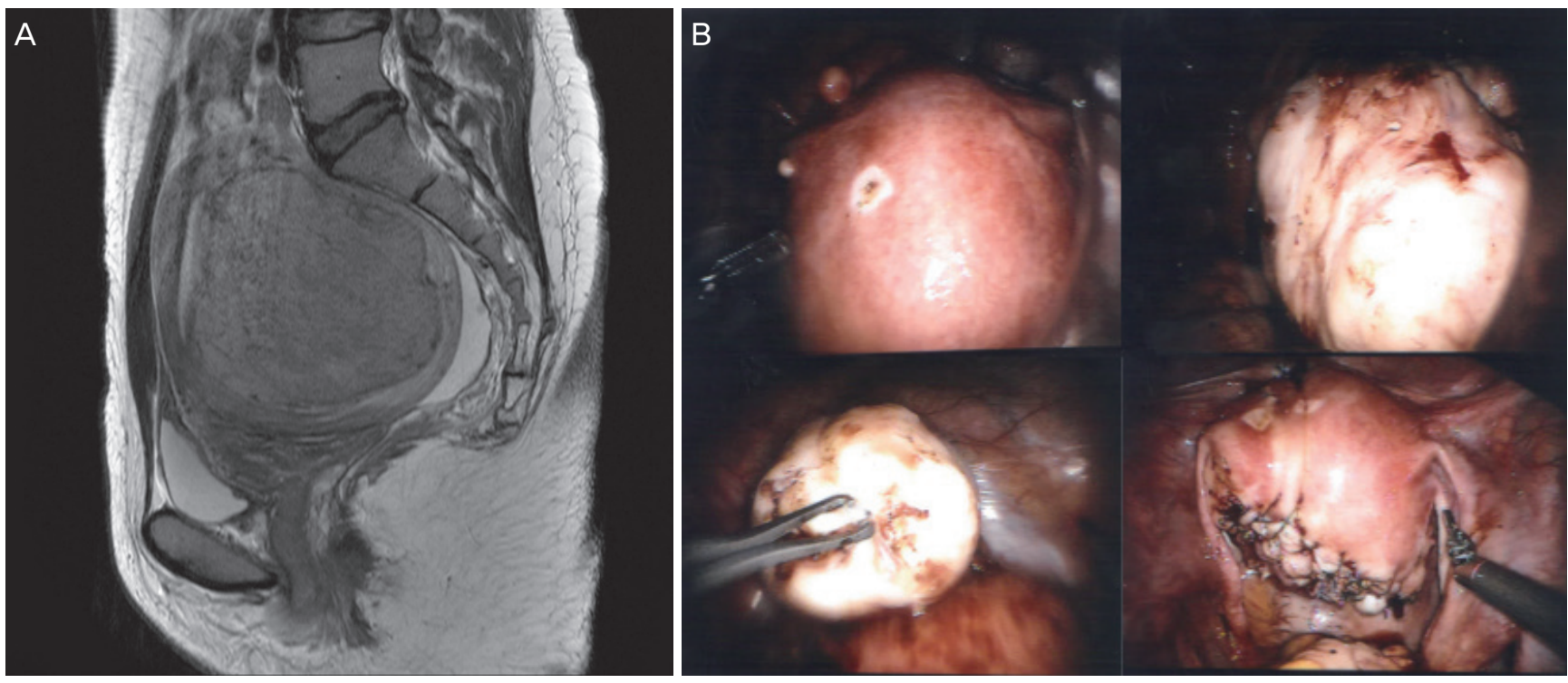

Fig. 3. Magnetic resonance imaging (MRI) and operative findings. (A) Sagittal T2-weighted MR images (T2WI) shows well-defined leiomyoma (arrows) with low signal intensity in posterior corpus of the uterus. This case was assigned as grade 3 in terms of SI of T2WI. (B) Huge myoma was extracted from the uterus and the uterine myometrium and serosa were sutured layer by layer for more than 3 layers using the da Vinci ${ }^{\circledR}$ Si Surgical System. 


\section{Obstetrics \& Gynecology Science}

So Yun Park, et al. Robotic myomectomy using MRI predictor

ceive [9].

Pitter et al. [10] investigated outcomes of pregnancy and parturition in 107 patients who underwent robotic myomectomy at 3 medical institutions. They observed that there were fewer cases of intra-abdominal adhesions during cesarean section (11.4\%), and one case had a rare occurrence of uterine rupture during pregnancy (1.1\%). Robotic myomectomy is reportedly advantageous even in cases of multiple myomas [3], when the SS technique is used $[4,11,12]$. Previous studies have revealed that SS robotic myomectomy has certain limitations including a longer duration of surgery and ergonomic challenges due to the semi-rigid, robotic instruments without wrists $[13,14]$.

After the development of a SS robotic myomectomy platform with wristed instruments, a recent multicenter retrospective analysis performed in the United States revealed no significant differences in EBL (83.3 mL vs. 109.2 $\mathrm{mL}, P=0.34$ ), duration of surgery (162.4 minutes vs. 162.4 minutes, $P=0.99$ ), frequency of overnight admission (odds ratio $[O R], 1.54 ; P=0.44)$, and post-operative complications $(\mathrm{OR}=1.3 ; P=0.78)$ between the SS and MS robotic myomectomy groups [15]. Our study demonstrated that there were no significant differences in the postoperative outcomes between both robotic surgical techniques (SS vs. MS). Furthermore, several studies have reported that most patients preferred a SS incision on the abdomen [16]. Thus, with respect to perioperative outcomes and cosmetic effect, SS robotic myomectomy is preferred over MS robotic myomectomy when available. In a previous study, we reported that SS robotic myomectomy is a feasible and safe procedure even in cases with large, multiple, and intramural type myomas [17] and SS robotic cystectomy can be used particularly in the advanced stages of endometriosis [18].

Since SS has a narrow range for maneuvering the instruments compared to MS, it is important to select which technique is suitable for a patient. Since there was no statistically significant difference between the surgical outcomes of SS and MS robotic myomectomy, the superiority of one technique over the other cannot be determined; hence, it is important to select the optimal surgical method according to each patient's uterine condition.

Pelvic MRI could be helpful in choosing between SS and MS before robotic myomectomy, since it is superior to conventional transvaginal sonography for ascertaining both, the correct embedment of the myoma in the uterine wall and the position of myoma, even in patients with 1-4 myomas and uterine volume $<375 \mathrm{~mL}$ [5]. In our study, when the fibroid was located deep within the uterine wall or the total diameter of myoma was large, robotic myomectomy was successfully performed using the MS method rather than the SS method.

Additionally, MRI also has an advantage of indicating the degree of stiffness or vascularity of the fibroid itself $[6,19]$, which is usually challenging using conventional transvaginal sonography. It is well known that there is a significantly inverse correlation between high $\mathrm{T} 2$ signal intensity of fibroids and their stiffness. Fibroids with high intensity have lower stiffness compared to those that have a low and homogenous signal intensity [6]. The results of this study also showed that myoma with a low T2 signal intensity is considerably stiffer, which thereby prolongs its resection time. Gadolinium-enhanced T1-weighted fat-suppressed sequences are important to confirm the vascularity of uterine myomas. Vascularity of myomas can vary between the cases; when the vascularity is high with strongly enhanced T1 image, the amount of blood loss during surgery might also be high. The stiffer the myomas, longer is the resection time, and more abundant the vascularity, higher is the blood loss during resection. Through this study, we confirmed that the degree of T2 signal intensity observed on pre-operative MRI correlates with the resection time during surgery.

Previous studies about magnetic resonance-guided focused ultrasound surgery reported that the signal intensity on T2weighted MR images is useful for selecting fibroids susceptible to thermal coagulation. Hypointense fibroids reportedly achieved more ablation volume than the hyperintense ones [20]. Myomas with low SI on T2-weighted MRI were more likely to reduce in size than those with high SI [21]. Based on this concept, we assumed that in uterine myomas with higher SI on MRI scans, care would be required for the control of bleeding during robotic myomectomy, especially, at the time of SS approach.

The fatal complication after myomectomy is uterine rupture in cases with a scarred uterus. Recent studies suggested that the risk of uterine rupture after laparoscopic myomectomy might vary depending on the characteristics of myomectomy and surgical techniques. The potential factors for uterine rupture were, entry in to the endometrial cavity and surgical techniques used to achieve uterine closure and hemostasis [22]. Bernardi et al. [23] reviewed 55 pregnancies that fol- 


\section{Obstetrics \& Gynecology Science}

Vol. 63, No. 6, 2020

lowed laparoscopic myomectomy. Uterine rupture occurred in patients with a short interval from surgery to conception ( $<12$ months), in those who underwent myomectomy for fibroids with large diameter $(>4 \mathrm{~cm})$, and in cases where the endometrial cavity had been entered during myomectomy. The rate of uterine rupture after robot-assisted myomectomy has been reported to be between $0.6 \%$ to $10 \%$. Huberlant et al. [8] showed the potential of robotic surgery in preserving the uterus and in reducing the obstetric complications. Indeed, endometrial breach were observed in only $15.1 \%$ of the cases, and no case of uterine rupture was observed. Intraoperative strategies including multi-layered uterine closure, avoidance of exposure to the endometrial cavity, and avoidance of excessive electro-cauterization to reduce devascularization were recommended to reduce the risk of uterine rupture during future pregnancies $[24,25]$. In our study, despite more than 3 layers of suturing, one patient developed uterine dehiscence at the site of a previous scar, where the uterine wall was thin. In terms of intra-operative, post-operative, and obstetric complications, more caution is required during robotic myomectomy when the myoma is huge and deep-seated, or if there are adhesions due to endometriosis or previous surgery. In Korean multicenter case series of uterine rupture in pregnancies following myomectomy, the authors suggested that pregnancy in women after laparoscopic or laparotomic myomectomy should be carefully observed and adequately counseled [26].

There are a few limitations of our study; first, our data is regarding an old system, namely da $\operatorname{Vinci}^{\circledR} \mathrm{Si}$, which will soon become redundant. The results from Xi and the new SP system might differ. Second, the follow-up period was not adequate to conclude the reproductive and obstetric outcomes. Finally, $80 \%$ of the patients who wanted to conceive had a successful conception after undergoing robotic myomectomy, and this finding was in line with those reported by previous studies, which reported that robotic myomectomy helped preserve fertility. However, the sample size of 15 patients was too small.

Robotic myomectomy is the procedure of choice for women with myomas who wish to preserve their fertility for future pregnancies. SS robotic myomectomy is an advanced technique to reduce pain and improve patient satisfaction. However, due to the limited area of the operative field, it is important to carefully choose between SS or MS technique for optimal surgical outcomes. Therefore, we emphasize on the need to assess the usefulness of preoperative MRI findings to select the optimal surgical technique for preservation of fertility. Larger the size and more the number of myomas or more deep-seated the myoma, more frequent is the tendency to select MS robotic myomectomy. Therefore, compared to SS, conventional MS approach is relevant for more complex robotic myomectomy.

Following a well-designed robotic myomectomy, the patients' menstruation and uterine endometrium can be normalized to achieve the restoration of fertility. Despite the limited range of maneuverability, SS robotic myomectomy might be feasible and safe for future pregnancy if the selection of the surgical technique based on preoperative MRI indicators is appropriate.

In conclusion, SS or MS robotic myomectomy can be recommended for patients with myoma who wish to plan a future pregnancy. However, the optimal surgical technique should be selected based on the preoperative MRI indicators, for achieving effective surgical outcomes and successful preservation of fertility. Further large clinical data is necessary to establish the detailed criteria for MRI indicators for selection of robotic myomectomy.

\section{Conflict of interest}

No potential conflict of interest relevant to this article was reported.

\section{Ethical approval}

This study was approved by the Institutional Review Board of Ewha Womans University Mokdong Hospital (No. 05-038-003).

\section{Patient consent}

Informed consent was obtained from all the study participants.

\section{References}

1. Stewart EA. Uterine fibroids. Lancet 2001;357:293-8. 


\section{Obstetrics \& Gynecology Science}

So Yun Park, et al. Robotic myomectomy using MRI predictor

2. Karlsen K, Mogensen O, Humaidana P, Kesmodel US, Ravn P. Uterine fibroids increase time to pregnancy: a cohort study. Eur J Contracept Reprod Health Care 2020;25:37-42.

3. Kim H, Shim S, Hwang $Y$, Kim M, Hwang H, Chung $Y$, et al. Is robot-assisted laparoscopic myomectomy limited in multiple myomas?: a feasibility for ten or more myomas. Obstet Gynecol Sci 2018;61:135-41.

4. Kim M, Kim MK, Kim ML, Jung YW, Yun BS, Seong SJ. Robotic single-site myomectomy: a single-center experience of 101 consecutive cases. Int J Med Robot 2019;15:e1959.

5. Dueholm M, Lundorf E, Hansen ES, Ledertoug S, Olesen $F$. Accuracy of magnetic resonance imaging and transvaginal ultrasonography in the diagnosis, mapping, and measurement of uterine myomas. Am J Obstet Gynecol 2002;186:409-15.

6. Jondal DE, Wang J, Chen J, Gorny KR, Felmlee J, Hesly G, et al. Uterine fibroids: correlations between MRI appearance and stiffness via magnetic resonance elastography. Abdom Radiol (NY) 2018;43:1456-63.

7. Wang T, Tang H, Xie Z, Deng S. Robotic-assisted vs. laparoscopic and abdominal myomectomy for treatment of uterine fibroids: a meta-analysis. Minim Invasive Ther Allied Technol 2018;27:249-64.

8. Huberlant S, Lenot J, Neron M, Ranisavljevic N, Letouzey $V$, De Tayrac R, et al. Fertility and obstetrical outcomes after robot-assisted laparoscopic myomectomy. Int J Med Robot 2020;16:e2059.

9. Pitter MC, Srouji SS, Gargiulo AR, Kardos L, SeshadriKreaden $U$, Hubert $H B$, et al. Fertility and symptom relief following robot-assisted laparoscopic myomectomy. Obstet Gynecol Int 2015;2015:967568.

10. Pitter MC, Gargiulo AR, Bonaventura LM, Lehman JS, Srouji SS. Pregnancy outcomes following robot-assisted myomectomy. Hum Reprod 2013;28:99-108.

11. Choi SH, Hong S, Kim M, Bae HS, Kim MK, Kim ML, et al. Robotic-assisted laparoscopic myomectomy: the feasibility in single-site system. Obstet Gynecol Sci 2019;62:56-64.

12. Lewis El, Srouji SS, Gargiulo AR. Robotic single-site myomectomy: initial report and technique. Fertil Steril 2015;103:1370-1377.e1.

13. Nam SH, Paek J, Choi C, Nam SH, Kim WY. A comparison between reduced-port robotic surgery and multiport robot-assisted laparoscopy for myomectomy. Eur J Obstet Gynecol Reprod Biol 2017;213:53-7.

14. Moawad GN, Tyan P, Paek J, Tappy EE, Park D, Choussein $S$, et al. Comparison between single-site and multiport robot-assisted myomectomy. J Robot Surg 2019;13:75764.

15. Goebel K, Goldberg JM. Women's preference of cosmetic results after gynecologic surgery. J Minim Invasive Gynecol 2014;21:64-7.

16. Yeung PP Jr, Bolden CR, Westreich D, Sobolewski C. Patient preferences of cosmesis for abdominal incisions in gynecologic surgery. J Minim Invasive Gynecol 2013;20:79-84.

17. Choi EJ, Rho AM, Lee SR, Jeong K, Moon HS. Robotic single-site myomectomy: clinical analysis of 61 consecutive cases. J Minim Invasive Gynecol 2017;24:632-9.

18. Moon HS, Shim JE, Lee SR, Jeong K. The comparison of robotic single-site surgery to single-port laparoendoscopic surgery for the treatment of advancedstage endometriosis. J Laparoendosc Adv Surg Tech A 2018;28:1483-8.

19. Maciel C, Tang YZ, Sahdev A, Madureira AM, Vilares Morgado P. Preprocedural MRI and MRA in planning fibroid embolization. Diagn Interv Radiol 2017;23:16371.

20. Park H, Yoon SW, Sokolov A. Scaled signal intensity of uterine fibroids based on T2-weighted MR images: a potential objective method to determine the suitability for magnetic resonance-guided focused ultrasound surgery of uterine fibroids. Eur Radiol 2015;25:3455-8.

21. Lénárd ZM, McDannold NJ, Fennessy FM, Stewart EA, Jolesz FA, Hynynen K, et al. Uterine leiomyomas: MR imaging-guided focused ultrasound surgery--imaging predictors of success. Radiology 2008;249:187-94.

22. Sutton C, Standen P, Acton J, Griffin C. Spontaneous uterine rupture in a preterm pregnancy following myomectomy. Case Rep Obstet Gynecol 2016;2016:6195621.

23. Bernardi TS, Radosa MP, Weisheit A, Diebolder H, Schneider U, Schleussner E, et al. Laparoscopic myomectomy: a 6-year follow-up single-center cohort analysis of fertility and obstetric outcome measures. Arch Gynecol Obstet 2014;290:87-91.

24. Buckley VA, Nesbitt-Hawes EM, Atkinson P, Won HR, Deans $R$, Burton $A$, et al. Laparoscopic myomectomy: 


\section{Obstetrics \& Gynecology Science}

Vol. 63, No. 6,2020

clinical outcomes and comparative evidence. J Minim Invasive Gynecol 2015;22:11-25.

25. Flyckt RL, Falcone T. Uterine rupture after laparoscopic myomectomy. J Minim Invasive Gynecol 2015;22:921-2.
26. Kim HS, Oh SY, Choi SJ, Park HS, Cho GJ, Chung JH, et al. Uterine rupture in pregnancies following myomectomy: a multicenter case series. Obstet Gynecol Sci 2016;59:454-62. 